\title{
REALIDADE VIRTUAL E PERCEPÇÃO AMBIENTAL: UM ESTUDO DE CASO DA PERCEPÇÃO DE ESPAÇO SOB USO DE ÓCULOS DE RV
}

\author{
BARROS, Bruno (1) \\ TONEO, Cícero (2) \\ (1) Universidade Federal de Pernambuco, Mestre \\ e-mail: barros bruno@hotmail.com \\ (2) Universidade Federal de Pernambuco, Graduando \\ e-mail: cicerotoneo@gmail.com
}

\begin{abstract}
RESUMO
O óculos de Realidade Virtual tem sido incipientemente utilizado como meio de divulgação de ambientes no mercado imobiliário e hoteleiro. Assim a Percepção Ambiental revela-se um elemento fundamental, uma vez que o ambiente virtual tem o objetivo de representar remota e fielmente um ambiente real. Sendo assim, este estudo teve o objetivo de verificar a eficácia dos óculos de realidade virtual como ferramenta remota de percepção ambiental. Para tanto, aplicou-se o método indutivo para determinar dados generalizáveis provenientes de estudos de caso. Como resultado, verificou-se um índice frágil de fidelidade entre as percepções de espaço em ambiente real e virtual.
\end{abstract}

Palavras chave: realidade virtual; percepção ambiental; espaço.

\begin{abstract}
The Virtual Reality glasses has been incipiently used as a means of publicizing environments in the real estate market and in the hotel business. Thus, Environmental Perception proves to be a fundamental element, since the virtual environment has the objective of remotely and faithfully representing a real environment. So, this study aimed to verify the effectiveness of virtual reality glasses as a remote tool for environmental perception. To do so, the inductive method was applied to determine generalizable data from case studies. As a result, there was a fragile fidelity index between the perceptions of space in real and virtual environment.
\end{abstract}

Keywords: virtual reality; environmental perception; space.

\section{INTRODUÇÃO}

A percepção ambiental tem como essência o discernimento do local em que se habita, com a exploração de todos os seus elementos e características, deste modo também é influenciada pelo repertório de conhecimento de cada indivíduo através de processos cognitivos, os quais envolvem conhecimento prévio, expectativas, necessidades e valores (PINHEIRO \& ELALI, 2011; PAIVA, SOBRAL \& VILLAROUCO, 2015; DEL RIO, 1999). Este processo ocorre através dos sentidos, como o da visão, de forma a estabelecer mecanismos de interface com a realidade ao decodificar os estímulos externos (OKAMOTO, 2002). 


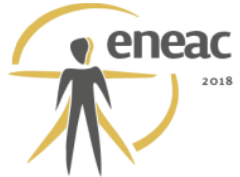

Entre a conexão homem/ ambiente, a reação para a realização de uma determinada tarefa ou atividade envolve a captação e o processamento das informações recebidas pelos diversos elementos que compõem o ambiente. Estas são questões cognitivas que envolvem as ações e reações realizadas pelo homem dentro de um determinado espaço (RANGEL $E$ MONT'ALVÃO, 2015). A concepção de ambientes engloba uma variedade sistemática de espaços desenvolvidos, edificados e ordenados, contemplando uma grande variedade de trabalhos humanos. Neste sentido, Vasconcelos, Villarouco e Soares (2009, p.7) destacam os aspectos psicológicos (sensações dos usuários captadas através da sua percepção sob o ambiente) como fundamento indispensável, além da associação com a estética e o limite espacial.

Com a evolução tecnológica contemporânea, o século XXI tem sido marcado pelo desenvolvimento de dispositivos multissensoriais que auxiliam as práticas de visualização de ambientes, buscando a aproximação entre um ambiente virtual e os usuários remotos das percepções do mundo real. Dentro deste contexto da percepção do entorno, despontam meios para aperfeiçoamento da relação usuário - ambiente virtual através de canais sensoriais.

Repousando o foco na Realidade Virtual (RV), verifica-se que os dispositivos multissensoriais permitem que o usuário interaja de modo imersivo com um ambiente tridimensional, o qual reflete de maneira real um artefato já concebido ou em fase de concepção (GERMANI et al., 2012; SHEHAB et al., 2010; UVA et al., 2010). A RV é comumente usada para delinear um conjunto de tecnologias que oferecem um ambiente virtual ao participante através de alguns dispositivos de visualização, dentre eles observa-se o óculos estereoscópico ou stereoglasses (WILSON, 1999; AKAGUI e KIRNER, 2004). Com efeito, sobre a percepção sensorial, incide este tipo de dispositivo, utilizado nas representações virtuais, provendo atributos da simulação da visualização de um artefato real ou imaginado através das respostas aos estímulos do usuário (FAGIANI et al., 2011; FITZSIMONS, 2012; DARDEN e SCHWARTZ, 2009).

Para que a transmissão simulada das informações visuais ocorra de maneira eficaz, faz-se necessário que o usuário tenha o mesmo nível de compreensão acerca dos dois ambientes, real e virtual. A relevância deste requisito se fundamenta no fato de que o ambiente virtual tem o objetivo de representar fielmente um ambiente real (ainda que imaginário).

O recurso do óculos estereoscópico de Realidade Virtual, comum e popularmente conhecido como óculos de Realidade Virtual, tem sido incipientemente utilizado como meio de divulgação de ambientes no mercado imobiliário e hoteleiro. Para o mercado imobiliário, a essência da motivação do uso desta tecnologia reside no fato de que o custo de decoração de um apartamento pequeno de alto padrão é de cerca de $R \$ 300$ mil, contra os $R \$ 80$ mil dos projetos em 3D mais sofisticados (DINIS e GULLINO, 2016). Os autores afirmam que as imobiliárias também viram vantagem em poder simular mais facilmente diversas configurações de um mesmo apartamento: trocar os móveis de lugar, por exemplo, e testar cores. Com efeito, o uso da RV no mercado imobiliário permite que o cliente visualize diversos empreendimentos simultaneamente sem se deslocar, poupando tempo em visitas a diversos estandes de venda. No que tange ao mercado hoteleiro, com o surgimento da possibilidade de se observar o ambiente em $360^{\circ}$, empresas como a Expedia investiram em 2016 mais de 1 bilhão de dólares nesse ramo, a qual já disponibiliza demonstrações em Realidade Virtual em suas principais plataformas de websites como Expedia.com, Hotels.com e Trivago, com o intuito de convidar seus usuários a visitar as acomodações oferecidas pelas plataformas (MUNDO VR, 2017).

No campo científico das investigações em Ergonomia (essencialmente na Ergonomia do Ambiente Construído), apesar de se observar uma etapa destinada à Percepção Ambiental (etapa constante na Metodologia Ergonômica para o Ambiente Construído), não se verificam sugestões, ou estudos, que estimulem tal análise com o apoio do óculos de realidade virtual. 
A lacuna associada ao processo ainda inicial de utilização científica do óculos de realidade virtual nos estimulou a enxergar a possibilidade de investigação da percepção visual ambiental por meio deste dispositivo, uma vez que acreditamos que o óculos de realidade virtual pode proporcionar uma percepção visual do ambiente tão eficaz quanto a percepção visual decorrente da própria presença física do indivíduo no espaço. Como recorte de pesquisa, selecionamos a percepção de espaço em termos de dimensão da área interna de ambientes. Para tanto, especificamos como estudo de caso uma brinquedoteca localizada dentro de uma universidade e utilizada para aulas experimentais com estudantes do curso de pedagogia. $O$ estudo abordou a percepção ambiental tanto para verificar limitações na percepção virtualizada, quanto para conduzir o acesso do usuário final a ambientes virtualizados eficazes. Sob procedimentos específicos de atuação, acreditamos que o óculos de realidade virtual poderia passar a atuar como ferramenta de apoio na verificação da percepção visual ambiental, o que viria a fornecer insumos teóricos para a elaboração de novas ferramentas voltadas à avaliação da percepção que tenham como base a realidade virtual.

\section{FUNDAMENTAÇÃO TEÓRICA}

Devido ao fato da RV ter sido criada há mais de duas décadas, sua definição acabou sendo modernizada, Kirner e Kirner (2011) afirmam que este fato deve-se aos aspectos tecnológicos mais atuais, como a multiplicidade de plataformas e a exequibilidade de softwares capazes de tratar elementos multisensoriais. Os autores afirmam que, o que antes se restringia a computadores de grande porte e a aplicações de computação gráfica, foi expandido para microcomputadores, plataformas móveis e Internet, envolvendo aplicações gráficas, sonoras, gestuais e de reação de tato e força. Desta forma, Kirner e Kirner (2011) definem a RV como uma técnica de interface computacional que considera o espaço tridimensional onde o usuário pode atuar de forma multisensorial, explorando aspectos deste espaço por meio da visão, audição e tato. Tendo em vista os pontos abordados pelos autores ao longo do tempo, é possível perceber que, de uma maneira geral, as definições de realidade virtual traçam referência a um processo de experiência através da imersão e interação baseadas em imagens gráficas tridimensionais, o que pode ser compreendido como uma simulação de um universo real ou imaginário produzido por computador.

O avanço das pesquisas na área vem melhorando a qualidade dos dispositivos de hardware, como capacetes de visualização, luvas e óculos mais leves e com mais recursos, o que contribui para despertar maior interesse dos vários segmentos industriais e aumentar a base de usuários e de aplicações no mundo todo. Em 2017, a grande popularização dos óculos de realidade virtual direcionados à reprodução da imagem através de smartphones tem estimulado as empresas a otimizarem esta tecnologia e oferecerem rapidamente os headsets ao mercado competitivo. Neste tipo de dispositivo, a tela do smartphone fica bem próxima dos olhos do usuário e cada um deles enxerga apenas metade da tela. Assim, um software específico força o envio de uma imagem distinta para cada olho, com uma sutil diferença de posicionamento, gerando a visão estereoscópica.

Diversos pesquisadores de realidade virtual concordam que, para ser considerada uma realidade virtual deve existir a ideia de imersão, interação e envolvimento (NETTO et al., 2002). Segundo Mendonça \& Mustaro 2011, é necessário que o indivíduo perceba que foi transportado psicologicamente para uma esfera virtual, para que sua imersão seja concreta. A sensação de imersão é obtida através de dispositivos como o Head Mounted Display (HMD) (BEGAULT, 1994; GRADESCKI, 1995; NETTO et al., 2002). As imagens a serem inseridas em óculos de RV devem ser do tipo esféricas, de 360 graus, e, para tanto, necessitam de duas câmeras com lentes fisheye (olho de peixe), cada câmera captura até 195ํㅡ a junção das imagens as quais as câmeras capturam são coladas e ajustadas através 


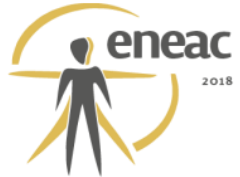

do software específico do dispositivo, criando em torno do usuário a representação do ambiente em vários ângulos (FREIRE, 2016).

A percepção psicológica de que o ambiente virtual realmente está em volta do usuário é a base da formação do que é a imersão em realidade virtual. $O$ aumento da imersão é diretamente proporcional ao crescimento do sentimento de presença dentro do ambiente proposto (MENDONÇA e MUSTARO, 2011). Nunes et al. (2011) alertam que muitas vezes pesquisadores da área de Computação concentram seus esforços no realismo no sentido da implementação e não avaliam a percepção de realismo que os usuários reais identificam em relação aos objetos e ferramentas. Os autores reforçam que o estudo de técnicas relacionadas à Psicologia e outras áreas que envolvem o comportamento humano pode colaborar com a compreensão desta percepção, contribuindo para a construção de ferramentas realistas, as quais tomem como foco a maneira como os usuários percebem este realismo proveniente dos ambientes virtuais.

A compreensão acerca das transmissões perceptivas entre ser humano e ambiente se revela como fator de suma importância para um entendimento mais profundo sobre a sociedade em vários tipos de circunstâncias as quais se insere. Ponto de partida de toda atividade humana, a percepção nos fornece integralmente as informações necessárias para a orientação em um ambiente, destacando-se a visão como sistema de percepção mais desenvolvido (BINS ELY, 2004). Neste sentido, é possível perceber que o ambiente pode influenciar o modo de como os indivíduos enxergam as situações cotidianas, uma vez que existe uma relação direta entre a conduta e o espaço, natural ou construído, ao qual está localizado.

O comportamento do indivíduo no espaço é gerado pelas suas particularidades e pela percepção do ambiente, que são comportamentos influenciados pelos estímulos ambientais (RANGEL E MONT'ALVÃO, 2015, p.04). A Percepção Ambiental traz estímulos, influencia as reações e o comportamento humano (VASCONCELOS, VILLAROUCO e SOARES, 2009). Bassani (2001) entende que a percepção ambiental é uma "experiência sensorial direta do ambiente em um dado momento, não sendo considerado um processo passivo de mera recepção e interpretação da estimulação ambiental pelas pessoas". Deste modo, esta área do conhecimento traduz-se como um aspecto fundamental na saúde e bem-estar do indivíduo, uma vez que ambientes mal projetados e com má qualidade física e espacial podem conduzir a uma insatisfação dos usuários, causando instabilidades emocionais e comportamentos destrutivos. As ações do homem sobre as influências ambientais são capazes de gerar diversas consequências, podendo interferir na qualidade de vida dos usuários (RHEINGANTZ, ALCANTARA e RIO, 2005).

Compreendemos um espaço através de um somatório de percepções e visões guardadas em nosso cérebro, que são arquivadas e construídas como imagens (MARQUES, 2006). A partir de informações visuais, o indivíduo é capaz de associar noção de espaço no ambiente (CHOI, 2001). Neste contexto, Azevedo (2016) destaca que a Percepção Espacial pode ser definida como uma maneira pela qual vivenciamos o espaço.

\section{METODOLOGIA}

O corrente estudo se alicerçou sob o método de abordagem Indutivo. Deste modo, nesta pesquisa, a partir do registro verbal de dois grupos de voluntários acerca da percepção do ambiente meio real e em meio virtual, pretende-se chegar a conclusões generalizáveis no que tange à percepção de espaço. Com isso, poderíamos gerar subsídios para verificação da eficácia da utilização do óculos de realidade virtual em substituição da necessidade de presença física dos indivíduos para percepção de ambientes. 


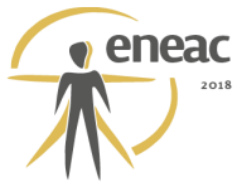

Como método de procedimento observou-se um perfil essencialmente comparativo, o qual se deu pelo cotejamento entre os dados obtidos a partir dos voluntários que observam o ambiente in loco e os voluntários que observam o ambiente em meio virtual.

O experimento foi realizado em uma Universidade, com estudantes da graduação em design (por ser o curso da Universidade cujos alunos possuem maior familiaridade com dimensões métricas de espaços). A pesquisa coletou o número de 140 voluntários (20\% dos alunos matriculados) e, a partir de então, foram formados dois grupos distintos de 70 estudantes. 0 primeiro grupo foi conduzido ao ambiente interno não familiar a estes usuários e pertencente à universidade, a brinquedoteca do curso de graduação em Pedagogia. O espaço contém $96 \mathrm{~m}^{2}$ e conta com diversos brinquedos, teatro de fantoches, piscina de bolinhas, banheiros para ambos os gêneros, tapetes em E.V.A, paredes coloridas em tons de baixa saturação, além de mesas e cadeiras. A Brinquedoteca está em atividade desde o primeiro semestre de 2015 e recebe visitas de crianças para interagir com o espaço, bem como ministrar aulas práticas a alunos de Pedagogia.

Figura 1: Brinquedoteca selecionada para estudo de caso.
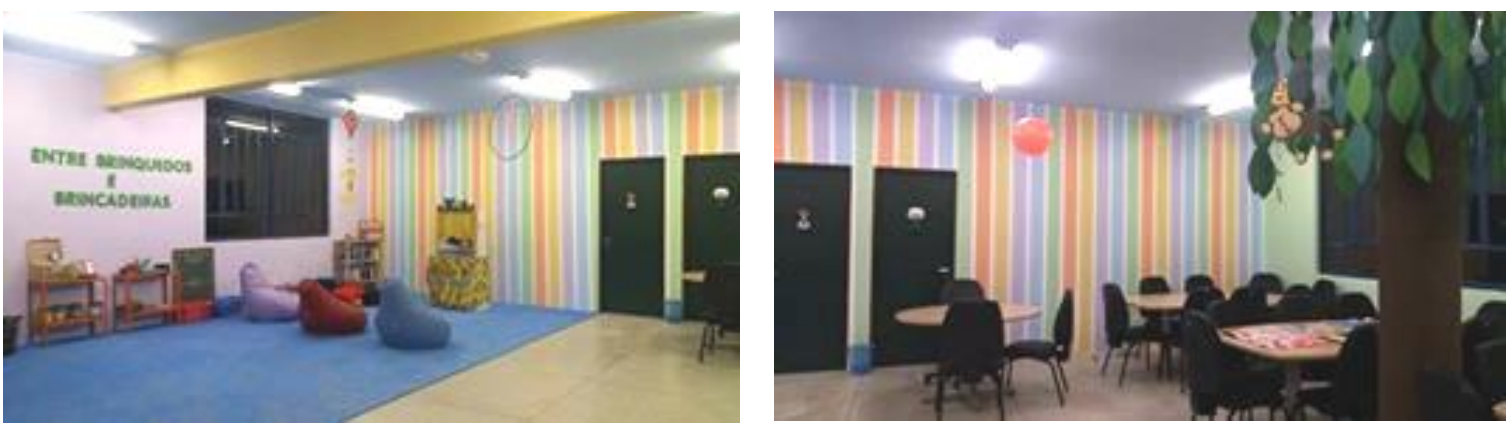

Fonte: Capturado pelos autores para a pesquisa.

$\mathrm{Na}$ brinquedoteca, um a um, os voluntários da pesquisa foram posicionados no centro do ambiente e tiveram 2 minutos para observarem todo o espaço. Em seguida, já fora da sala, cada voluntário descreveu (em papel) a dimensão métrica percebida acerca do ambiente ora observado. O segundo grupo de voluntários foi conduzido a uma sala de aula tradicional do curso de Design, onde (um a um) vestiram o óculos de realidade virtual e, no período de 2 minutos, observaram em meio virtual o mesmo ambiente ao qual o grupo 1 foi exposto. Em seguida, cada voluntário deste grupo 2 registrou em papel a dimensão do ambiente observado em meio virtual.

A ação de cada voluntário de ambos os grupos foi individual, não foram permitidas observações em grupo, tampouco descrições grupais acerca do ambiente. Os membros de cada grupo foram distintos, não foi permitida a participação de um mesmo voluntário em ambos os grupos. Também não se permitiu a participação de voluntários que já conheciam - ambiente da brinquedoteca, pois se buscou a ausência de familiaridade através da homogeneização das condições de ineditismo na percepção.

A imagem $360^{\circ}$ da brinquedoteca foi capturada com uma câmera de duas lentes olho de peixe do modelo Pano 360 , posicionada no centro da sala com tripé à altura de $1,60 \mathrm{~m}$, exatamente no local onde os voluntários foram dispostos para observação in loco. O óculos de RV utilizado foi do tipo cellular holder do modelo VR Box, onde se inseriu a imagem $360^{\circ}$ em um aparelho smartphone de tela de 5,5" da marca Sansung. Com isso, o dispositivo foi encaixado no óculos e a visualização em meio virtual pôde ser realizada. 


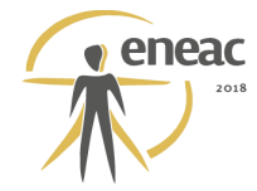

Figura 2: Modelo de câmera duplo olho de peixe e óculos RV utilizado na pesquisa.
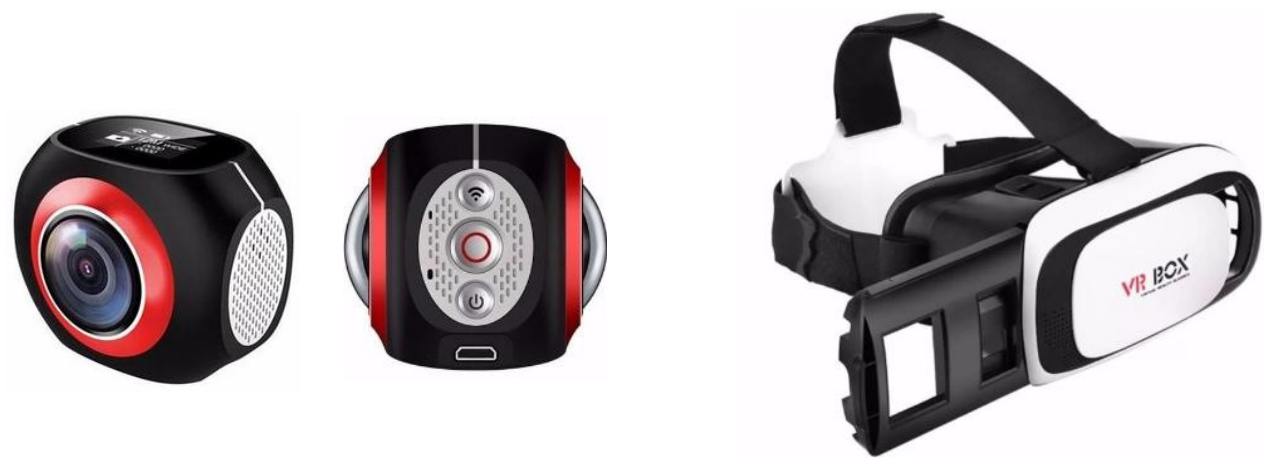

Fonte: Capturado pelos autores para a pesquisa.

Por fim, o índice de fidelidade das dimensões métricas foi identificado e comparado entre os dois grupos, no intuito de verificar o nível de eficácia do uso do óculos de realidade virtual na identificação da percepção de espaço.

\section{RESULTADOS}

O Grupo 1, contendo 70 voluntários, foi direcionado para observação presencial do ambiente real (físico). As principais características do Grupo 1 foi sua composição eclética de faixa etária e proporção quase igualitária aos gêneros. O grupo foi composto de pessoas entre 17 a 40 anos de idade contendo em maior parcela indivíduos do sexo masculino. Cerca de $77 \%$ do grupo foi composto por pessoas entre 17 a 24 anos de idade contendo em sua maioria indivíduos do sexo feminino correspondendo à $41,35 \%$ e $35,65 \%$ correspondente ao sexo masculino. Para os voluntários com faixa-etária entre 25 a 40 anos de idade, o gênero feminino foi composto por $7 \%$ e o gênero masculino correspondeu a $16 \%$. O número total de voluntários do gênero masculino soma 36 pessoas, índice referente a $51,65 \%$ de todos voluntários do grupo 1 .

Para uma melhor visualização das respostas, foi elaborado um quadro correspondente aos palpites dos voluntários acerca da área por metro quadrado.

Tabela 1: Palpites de dimensão métrica provenientes dos voluntários em ambiente real.

\begin{tabular}{ccc}
\hline Área por $\mathbf{m}^{2}$ & Número de Pessoas & Porcentagem \\
\hline $4 \mathrm{~m}^{2}$ à $20 \mathrm{~m}^{2}$ & 14 & $20 \%$ \\
\hline $21 \mathrm{~m}^{2}$ à $40 \mathrm{~m}^{2}$ & 13 & $18,58 \%$ \\
\hline $41 \mathrm{~m}^{2}$ à $60 \mathrm{~m}^{2}$ & 14 & $20 \%$ \\
\hline $61 \mathrm{~m}^{2}$ à $80 \mathrm{~m}^{2}$ & 11 & 15,71 \\
\hline $81 \mathrm{~m}^{2}$ à $100 \mathrm{~m}^{2}$ & 7 & $10 \%$ \\
\hline $101 \mathrm{~m}^{2}$ à $120 \mathrm{~m}^{2}$ & 1 & $1,42 \%$ \\
\hline $121 \mathrm{~m}^{2}$ à $140 \mathrm{~m}^{2}$ & 0 & $0 \%$ \\
\hline $141 \mathrm{~m}^{2}$ à $160 \mathrm{~m}^{2}$ & 2 & $2,86 \%$ \\
\hline $161 \mathrm{~m}^{2}$ à $180 \mathrm{~m}^{2}$ & 1 & $1,42 \%$ \\
\hline $181 \mathrm{~m}^{2}$ à $200 \mathrm{~m}^{2}$ & 2 & $2,86 \%$ \\
\hline $201 \mathrm{~m}^{2}$ à $300 \mathrm{~m}^{2}$ & 3 & $4,30 \%$ \\
\hline $301 \mathrm{~m}^{2}$ à $400 \mathrm{~m}^{2}$ & 0 & $0 \%$ \\
\hline $401 \mathrm{~m}^{2}$ à $500 \mathrm{~m}^{2}$ & 1 & $1,42 \%$ \\
\hline Acima de $500 \mathrm{~m}^{2}$ & 1 & $1,42 \%$
\end{tabular}

Fonte: Elaborado pelos autores para a pesquisa. 
Foi possível perceber que mais de $58 \%$ dos voluntários que visitaram o ambiente real acreditam que o ambiente tenha de $4 \mathrm{~m}^{2}$ a $60 \mathrm{~m}^{2}$, apenas $10 \%$ dos voluntários conseguiram se aproximar do valor exato por metro quadrado da brinquedoteca, $96 \mathrm{~m}^{2}$. Cabe lembrar que o objetivo da pesquisa não foi focar nas pessoas que acertaram as medidas corretas do ambiente, mas sim comparar essa percepção com a do segundo grupo, para certificar se as percepções no ambiente real e virtual se manifestam da mesma maneira.

O Grupo 2, também contendo 70 voluntários, foi conduzido para a análise do ambiente virtual através do óculos RV. Com a captura da imagem 360 da brinquedoteca os voluntários sentiam-se imersos naquela realidade e puderam, através do que foi observado, palpitar acerca da dimensão do espaço. As principais características do Grupo 2 é sua composição de 58,60\% de voluntários do gênero feminino e a faixa-etária entre 17 e 51 anos de idade. Por volta de $64,30 \%$ do grupo foi constituído por pessoas entre 17 e 24 anos de idade contendo em sua maioria indivíduos do gênero feminino correspondendo à $40,40 \%$ e $24,30 \%$ referente ao sexo masculino. Para os voluntários com faixa-etária entre 25 e 51 anos de idade o menor número de pessoas é do gênero masculino equivalendo a $17,14 \%$ e $18,60 \%$ do restante são indivíduos do gênero feminino. Somando o número de voluntários do gênero masculino do grupo 2 obtemos o total de 29 pessoas, índice correspondente à $41,40 \%$.

Também para uma melhor visualização dos resultados, os dados do grupo 2 foram inseridos em um quadro, respeitando do menor até o maior palpite por metro quadrado.

Tabela 2: Palpites de dimensão métrica provenientes dos voluntários em ambiente virtual.

\begin{tabular}{ccc}
\hline Área por $\mathbf{m}^{2}$ & Número de Pessoas & Porcentagem \\
\hline $4 \mathrm{~m}^{2}$ à $20 \mathrm{~m}^{2}$ & 17 & $24,30 \%$ \\
\hline $21 \mathrm{~m}^{2}$ à $40 \mathrm{~m}^{2}$ & 19 & $27,14 \%$ \\
\hline $41 \mathrm{~m}^{2}$ à $60 \mathrm{~m}^{2}$ & 12 & $17,14 \%$ \\
\hline $61 \mathrm{~m}^{2}$ à $80 \mathrm{~m}^{2}$ & 6 & $8,60 \%$ \\
\hline $81 \mathrm{~m}^{2}$ à $100 \mathrm{~m}^{2}$ & 6 & $8,60 \%$ \\
\hline $101 \mathrm{~m}^{2}$ à $120 \mathrm{~m}^{2}$ & 4 & $5,71 \%$ \\
\hline $121 \mathrm{~m}^{2}$ à $140 \mathrm{~m}^{2}$ & 0 & $0 \%$ \\
\hline $141 \mathrm{~m}^{2}$ à $160 \mathrm{~m}^{2}$ & 3 & $4,30 \%$ \\
\hline $161 \mathrm{~m}^{2}$ à $180 \mathrm{~m}^{2}$ & 0 & $0 \%$ \\
\hline $181 \mathrm{~m}^{2}$ à $200 \mathrm{~m}^{2}$ & 3 & $4,30 \%$ \\
\hline
\end{tabular}

Fonte: Elaborado pelos autores para a pesquisa.

Diferentemente do grupo 1, verificou-se que o maior palpite foi equivalente a 200 metros quadrados, desse modo, as quatro últimas linhas (201 a 300m²; 301 a $400 \mathrm{~m}^{2} ; 401$ a $500 \mathrm{~m}^{2}$; e acima de $500 \mathrm{~m}^{2}$ ) foram eliminadas da tabela por não conter palpites referente a tais valores. Observou-se ainda que $69,60 \%$ dos voluntários que visitaram a brinquedoteca em ambiente virtual através do óculos de realidade virtual acreditam que o ambiente possua de 4 a 60 metros quadrados. Dentre os voluntários que palpitaram que o ambiente virtual possui entre 81 a $100 \mathrm{~m}^{2}$, apenas duas pessoas conseguiram resultados aproximados das medidas do ambiente real, os dois voluntários palpitaram que o ambiente possuía $96 \mathrm{~m}^{2}$.

\subsection{Análise comparativa dos dados}

Em posse dos dados individuais obtidos acerca do ambiente em meio real, através de somatório e divisão aritmética, foi possível calcular a área média percebida pelos voluntários para o espaço. Da forma, os dados dimensionais individuais colhidos acerca do meio virtual também foram submetidos a somatório e divisão aritmética, revelando as áreas dispostas no quadro que se segue: 


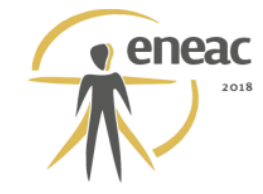

Tabela 3: Palpites e média aritmética para os ambientes real e virtual.

\begin{tabular}{|c|c|c|}
\hline & $\begin{array}{r}\text { Area do ambiente } \\
\text { em meio real }\end{array}$ & $\begin{array}{l}\text { Area do ambiente } \\
\text { em meio virtual }\end{array}$ \\
\hline 1. & $80 \mathrm{~m}^{2}$ & $42 \mathrm{~m}^{2}$ \\
\hline 2. & $11 \mathrm{~m}^{2}$ & $15 \mathrm{~m}^{2}$ \\
\hline 3. & $8 \mathrm{~m}^{2}$ & $100 \mathrm{~m}^{2}$ \\
\hline 4. & $288 m^{2}$ & $20 \mathrm{~m}^{2}$ \\
\hline 5. & $60 \mathrm{~m}^{2}$ & $40 \mathrm{~m}^{2}$ \\
\hline 6. & $80 \mathrm{~m}^{2}$ & $15 \mathrm{~m}^{2}$ \\
\hline 7. & $4 \mathrm{~m}^{2}$ & $25 \mathrm{~m}^{2}$ \\
\hline 8. & $100 \mathrm{~m}^{2}$ & $200 \mathrm{~m}^{2}$ \\
\hline 9. & $500 \mathrm{~m}^{2}$ & $24 \mathrm{~m}^{2}$ \\
\hline 10. & $56 \mathrm{~m}^{2}$ & $96 \mathrm{~m}^{2}$ \\
\hline 11. & $50 \mathrm{~m}^{2}$ & $96 \mathrm{~m}^{2}$ \\
\hline 12. & $42 \mathrm{~m}^{2}$ & $33 \mathrm{~m}^{2}$ \\
\hline 13. & $88 \mathrm{~m}^{2}$ & $200 \mathrm{~m}^{2}$ \\
\hline 14. & $80 \mathrm{~m}^{2}$ & $104 \mathrm{~m}^{2}$ \\
\hline 15. & $28 \mathrm{~m}^{2}$ & $100 \mathrm{~m}^{2}$ \\
\hline 16. & $12 \mathrm{~m}^{2}$ & $50 \mathrm{~m}^{2}$ \\
\hline 17. & $42 \mathrm{~m}^{2}$ & $22 \mathrm{~m}^{2}$ \\
\hline 18. & $54 \mathrm{~m}^{2}$ & $20 \mathrm{~m}^{2}$ \\
\hline 19. & $20 \mathrm{~m}^{2}$ & $48 \mathrm{~m}^{2}$ \\
\hline 20. & $48 \mathrm{~m}^{2}$ & $150 \mathrm{~m}^{2}$ \\
\hline 21. & $48 \mathrm{~m}^{2}$ & $6 \mathrm{~m}^{2}$ \\
\hline 22. & $20 \mathrm{~m}^{2}$ & $48 \mathrm{~m}^{2}$ \\
\hline 23. & $160 \mathrm{~m}^{2}$ & $36 \mathrm{~m}^{2}$ \\
\hline 24. & $30 \mathrm{~m}^{2}$ & $48 \mathrm{~m}^{2}$ \\
\hline 25. & $30 \mathrm{~m}^{2}$ & $120 \mathrm{~m}^{2}$ \\
\hline 26. & $8 \mathrm{~m}^{2}$ & $15 \mathrm{~m}^{2}$ \\
\hline 27. & $94,5 \mathrm{~m}^{2}$ & $60 \mathrm{~m}^{2}$ \\
\hline 28. & $42 \mathrm{~m}^{2}$ & $24 \mathrm{~m}^{2}$ \\
\hline 29. & $30 \mathrm{~m}^{2}$ & $35 \mathrm{~m}^{2}$ \\
\hline 30. & $300 \mathrm{~m}^{2}$ & $20 \mathrm{~m}^{2}$ \\
\hline 31. & $48 \mathrm{~m}^{2}$ & $100 \mathrm{~m}^{2}$ \\
\hline 32. & $12 \mathrm{~m}^{2}$ & $70 \mathrm{~m}^{2}$ \\
\hline 33. & $10 \mathrm{~m}^{2}$ & $30 \mathrm{~m}^{2}$ \\
\hline 34. & $48 \mathrm{~m}^{2}$ & $100 \mathrm{~m}^{2}$ \\
\hline 35. & $64 \mathrm{~m}^{2}$ & $48 \mathrm{~m}^{2}$ \\
\hline 36. & $80 \mathrm{~m}^{2}$ & $25 \mathrm{~m}^{2}$ \\
\hline 37. & $64 \mathrm{~m}^{2}$ & $12 \mathrm{~m}^{2}$ \\
\hline 38. & $100 \mathrm{~m}^{2}$ & $80 \mathrm{~m}^{2}$ \\
\hline 39. & $6 \mathrm{~m}^{2}$ & $15 \mathrm{~m}^{2}$ \\
\hline 40. & $108 \mathrm{~m}^{2}$ & $24 \mathrm{~m}^{2}$ \\
\hline 41. & $64 \mathrm{~m}^{2}$ & $9 \mathrm{~m}^{2}$ \\
\hline 42. & $200 \mathrm{~m}^{2}$ & $120 \mathrm{~m}^{2}$ \\
\hline 43. & $28 \mathrm{~m}^{2}$ & $150 \mathrm{~m}^{2}$ \\
\hline 44. & $30 \mathrm{~m}^{2}$ & $15 \mathrm{~m}^{2}$ \\
\hline 45. & $8 \mathrm{~m}^{2}$ & $112 \mathrm{~m}^{2}$ \\
\hline 46. & $24 \mathrm{~m}^{2}$ & $200 \mathrm{~m}^{2}$ \\
\hline 47. & $40 \mathrm{~m}^{2}$ & $40 \mathrm{~m}^{2}$ \\
\hline 48. & $35 \mathrm{~m}^{2}$ & $80 \mathrm{~m}^{2}$ \\
\hline 49. & $5 \mathrm{~m}^{2}$ & $80 \mathrm{~m}^{2}$ \\
\hline 50. & $48 \mathrm{~m}^{2}$ & $24 \mathrm{~m}^{2}$ \\
\hline 51. & $60 \mathrm{~m}^{2}$ & $36 \mathrm{~m}^{2}$ \\
\hline 52. & $96 \mathrm{~m}^{2}$ & $6 \mathrm{~m}^{2}$ \\
\hline 53. & $300 \mathrm{~m}^{2}$ & $48 \mathrm{~m}^{2}$ \\
\hline 54. & $180 \mathrm{~m}^{2}$ & $20 \mathrm{~m}^{2}$ \\
\hline
\end{tabular}




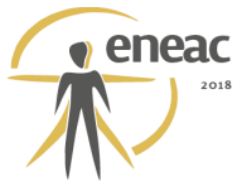

\begin{tabular}{|r|r|r|}
\hline 55. & $48 \mathrm{~m}^{2}$ & $40 \mathrm{~m}^{2}$ \\
\hline 56. & $80 \mathrm{~m}^{2}$ & $17 \mathrm{~m}^{2}$ \\
\hline 57. & $80 \mathrm{~m}^{2}$ & $4 \mathrm{~m}^{2}$ \\
\hline 58. & $6 \mathrm{~m}^{2}$ & $150 \mathrm{~m}^{2}$ \\
\hline 59. & $35 \mathrm{~m}^{2}$ & $6 \mathrm{~m}^{2}$ \\
\hline 60. & $70 \mathrm{~m}^{2}$ & $70 \mathrm{~m}^{2}$ \\
\hline 61. & $40 \mathrm{~m}^{2}$ & $24 \mathrm{~m}^{2}$ \\
\hline 62. & $150 \mathrm{~m}^{2}$ & $60 \mathrm{~m}^{2}$ \\
\hline 63. & $200 \mathrm{~m}^{2}$ & $60 \mathrm{~m}^{2}$ \\
\hline 64. & $30 \mathrm{~m}^{2}$ & $21 \mathrm{~m}^{2}$ \\
\hline 65. & $5 \mathrm{~m}^{2}$ & $40 \mathrm{~m}^{2}$ \\
\hline 66. & $70 \mathrm{~m}^{2}$ & $48 \mathrm{~m}^{2}$ \\
\hline 67. & $48 \mathrm{~m}^{2}$ & $64 \mathrm{~m}^{2}$ \\
\hline 68. & $73 \mathrm{~m}^{2}$ & $15 \mathrm{~m}^{2}$ \\
\hline 69. & $96 \mathrm{~m}^{2}$ & $50 \mathrm{~m}^{2}$ \\
\hline 70. & $100 \mathrm{~m}^{2}$ & $35 \mathrm{~m}^{2}$ \\
\hline média & $\mathbf{7 3 , 2 7 8 5 7 1 4 3 \mathrm { m } ^ { 2 }}$ & $\mathbf{5 6 , 5 7 1 4 2 8 5 7 \mathrm { m } ^ { 2 }}$ \\
\hline
\end{tabular}

Fonte: Elaborado pelos autores para a pesquisa.

O valor médio para o ambiente visualizado em meio físico real totalizou $73,28 \mathrm{~m}^{2}$. Tal valor obtido não previu área real do espaço, que é de $96 \mathrm{~m}^{2}$, contudo, nossa intenção foi a da verificação de similaridades nas percepções entre os meios de visualização utilizados. Em se tratando do meio virtual, o valor de área média identificado de $56,57 \mathrm{~m}^{2}$, o qual não só se distanciou ainda mais do valor real da área do ambiente, como também apresentou uma diferença de $16,71 \mathrm{~m}^{2}$ do valor percebido em ambiente físico real.

A pesquisa nos leva a acreditar que, ao visualizar a reprodução de ambientes em modo virtual, os espaços ficam aparentemente menores do que são em meio físico real. Neste estudo, a redução dimensional de $16,71 \mathrm{~m}^{2}$ equivaleu à uma percepção da brinquedoteca $22.8 \%$ menor que a real. Deste modo, em se tratando de fotografias $360^{\circ}$ inseridas em smartphone para visualização em realidade virtual através de óculos RV, este estudo nos fornece indícios para desaconselhar o uso desta tecnologia na percepção de espaço em substituição da presença física do ser humano.

\section{CONCLUSÕES}

O processo inicial de estruturação desta pesquisa se alicerçou na hipótese de que as percepções de espaço real e virtual (com uso de fotografia e óculos de realidade virtual) seriam similares, o que nos levaria a indicar a utilização desta tecnologia em substituição da presença física do indivíduo para a percepção de espaço. O resultado do estudo provou o contrário, ficou nítida a diferença entre as percepções, resultando em uma percepção de espaço de dimensões inferiores em meio virtual.

Neste estudo, a dimensão da diferença entre as áreas $\left(16,71 \mathrm{~m}^{2}\right)$ pode equivaler a um cômodo residencial, trata-se de uma área não percebida de dimensão considerável. Dentro deste contexto, lembramos que o mercado imobiliário e hoteleiro já lançam mão da RV por meio do óculos para apresentar remotamente para clientes espaços para locação ou venda. Tais espaços podem estar sendo visualizados dimensionalmente menores do que realmente são. Esta falha na fidelização entre o real e o virtual pode estar promovendo uma apresentação ineficaz dos ambientes e, com isso, implicando em frustração e arrependimento acerca da aquisição de um imóvel ou escolha de hospedagem para um período de férias. Este mesmo tipo de desvantagem também pode estar sendo ocorrendo no universo da visualização de ambientes internos de museus e obras de arte remotas. 


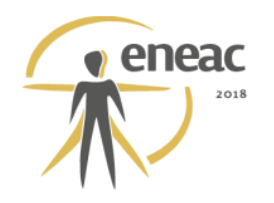

Ao verificar a falha nos índices de fidelização na percepção ambiental de espaço entre o real e o virtual, esta pesquisa fornece um alerta à sociedade quanto ao uso e avaliação exclusivamente remota de espaços. Os aspectos de frustração e arrependimento na aquisição de imóvel ou reservas em hotéis, citados anteriormente, podem demandar modificações e reformas desnecessárias nas edificações, as quais foram baseadas em um espaço de dimensões perceptualmente diferentes.

Os resultados ora encontrados e descritos nesta publicação apontam para a necessidade de uma ampliação neste estudo, aumentando a amostra de voluntários, bem como diversificando o perfil destes. No momento, para o âmbito da Percepção Ambiental na Ergonomia, alertamos para esta possível ineficácia na utilização do óculos de realidade virtual em substituição da necessidade de presença física dos indivíduos para percepção de espaço. A verificação ergonômica do modo como os usuários percebem o ambiente precisa ser uma experiência real e completa, uma vez que promove o estabelecimento de recomendações de modificação e adaptação do ambiente de acordo com as características e limitações dos usuários.

A evolução tecnológica tem permitido que o óculos de realidade virtual venha sendo utilizado com eficácia em diversas áreas de atuação. Acreditamos que, com avanço tecnológico, associado a pesquisas científicas que busquem identificação de falhas e aprimoramento de sistemas, o óculos de RV possa vir a atuar como ferramenta de apoio na verificação da percepção visual ambiental.

\section{REFERÊNCIAS BIBLIOGRÁFICAS}

AKAGUI, Daniela; KIRNER, Cláudio. Desenvolvimento de Aplicações de Realidade Aumentada com ARToolKit. In: WRA - Workshop de Realidade Aumentada. Anais... 2004. Piracicaba, SP. P.6-8.

AZEVEDO, Lino. Psicomotricidade: Percepção Espacial. Youtube, 2016. Disponível em: <https://www.youtube.com/watch?v=u2CGAA-BPbg> acesso em: 14 de novembro de 2017.

BASSANI, Marlise A. Psicologia Ambiental: Contribuições para a Educação Ambiental. In: HAMMES, Valéria S. (Org.). Educação Ambiental para o Desenvolvimento Sustentável Proposta Metodológica de Macroeducação. São Paulo, 2004. v.2, p. 153-157.

BEGAULT, D. R. 3-D Sound for virtual reality and multImedia. Academic Press, Cambridge, MA, 1994.

BINS ELY, V. H. Acessibilidade Espacial: Condições necessária para o projeto de ambientes inclusivos. In: MORAES, Anamaria de (Org.). Ergonomia do ambiente construído e habitado: ambiente urbano, ambiente público, ambiente laboral. Rio de Janeiro: iUsEr, 2004, p. 17-40.

$\mathrm{CHOI}, \mathrm{J}$. Sex Differences in spatial abilities in humans: two levels of explanation. In: VOKEY, J. R.; ALLEN, S. W. Psychological Sketches. 5. ed. Lethbridge: Department of Psychology and Neuroscience, University of Lethbridge, 2001.

DARDEN, M. A.; SCHWARTZ, C. J. Investigation of skin tribology and its effects on the tactile attributes of polymer fabrics. Wear, v. 267, n. 5-8, p. 1289-1294, 2009.

DEL RIO, V. Cidade da Mente, Cidade Real: Percepção Ambiental e Revitalização na Área Portuária do RJ. In: DEL RIO, Vicente; OLIVEIRA, Lívia (Orgs). Percepção ambiental: A experiência brasileira, p. 3-22, 2ªํㅡ. ed. São Paulo: Studio Nobel, 1999.

DINIS, Ana Carolina; GULLINO, Daniel. Construtoras apostam em óculos de realidade virtual - tecnologia facilita visita dos clientes aos imóveis. O Globo, 2016. Disponível em: 


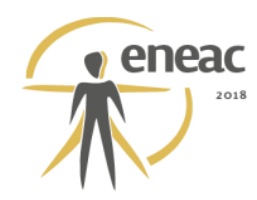

https://oglobo.globo.com/economia/construtoras-apostam-em-oculos-de-realidade-virtual19303530. Acesso em 26 de junho de 2017.

FAGIANI, R.; MASSI, F.; CHATELET, E.; BERTHIER, Y.; AKAY, A. Tactile perception by friction induced vibrations. Tribology International, v. 44, n. 10, p. 1100-1110, 2011.

FITZSIMONS, J. K. Seeing Motion Otherwise: Architectural Design and the Differently Sensing and Mobile. Space and Culture, v. 15, n. 3, p. 239-257, 2012.

FREIRE, Raquel. Entenda como funciona uma câmera 360 graus. Techtudo, 2016. Disponível em: <http://www.techtudo.com.br/noticias/noticia/2016/10/entenda-comofunciona-uma-camera-360-graus.html> acesso em: 13 de outubro de 2017.

GERMANI, M.; MENGONI, M.; PERUZZINI, M. An approach to assessing virtual environments for synchronous and remote collaborative design. Advanced Engineering Informatics, v. 26, n. 4, p. $793-813,2012$.

GRADESCKI, J. The virtual reality construction kit. John Wiley \& Sons, 340 Pp., 1995.

KIRNER, C.; KIRNER, T. G. Evolução e Tendências da Realidade Virtual e da Realidade Aumentada. In: RIBEIRO, M. ZORZAL, E. (Orgs). Realidade Virtual e Aumentada: aplicações e tendências. Ed. SBC - Sociedade Brasileira de Computação, Uberlândia-MG, 2011, p. 10-25.

MARQUES. Jorge. As imagens do desenho: Percepção espacial e representação. Faculdade de Belas Artes da Universidade do Porto, 2006.

MENDONÇA, R. L.; MUSTARO, P. N. Como tornar plicações de realidade virtual e aumentada, ambientes virtuais e sistemas de realidade mista mais imersivos. In: RIBEIRO, M. ZORZAL, E. (Orgs). Realidade Virtual e Aumentada: aplicações e tendências. Ed. SBC - Sociedade Brasileira de Computação, Uberlândia-MG, 2011, p. 96-110.

MUNDO VR. Expedia convida a visitar hotéis em Realidade Virtual antes de reservar. Mundo VR - 14 de abril de 2017. Disponível em: http://mundovr.com.br/2017/04/14/expediaconvida-a-visitar-hoteis-em-realidade-virtual-antes-de-reservar/. Acesso em: 26 de junho de 2017.

NETTO, Antônio. MACHADO, Liliane. OLIVEIRA, Maria. Realidade Virtual - Definições, Dispositivos e Aplicações. Instituto de Ciências Matemáticas e de Computação - ICMC, 2002. Universidade de São Paulo - USP.

NUNES, F. L. S.; COSTA, R. M. E. M.; MACHADO, L. S.; MORAES, R. M. Desenvolvendo aplicações de RVA para a saúde: imersão, realismo e motivação. In: RIBEIRO, M. ZORZAL, E. (Orgs). Realidade Virtual e Aumentada: aplicações e tendências. Ed. SBC - Sociedade Brasileira de Computação, Uberlândia-MG, 2011, p. 82-95.

OKAMOTO, J. Percepção ambiental e comportamento: visão holística da percepção ambiental na arquitetura e na comunidade. São Paulo: Editora Mackenzie, 2002.

PAIVA, M. M. B.; SOBRAL, E. R. A.; VILLAROUCO, V. The elderly and environmental perception in collective housing. In: AHFE 2015: 6th International Conference on Applied Human Factors and Ergonomics 2015 and the Affiliated Conferences. Procedia Manufacturing 3, 2015. p. 6505-6512.

PINHEIRO, J. Q.; ELALI, G. A.. Comportamento Socioespacial Humano. In: Temas básicos em Psicologia Ambiental. CAVALCANTE, Sylvia; ELALI, Gleice A. (Orgs). Petrópolis: Editora Vozes, 2011.

RANGEL, M. M.; MONT'ALVÃO, C. A observação do comportamento do usuário para o wayfinding no ambiente construído. Estudos em Design. v.23, n.3, p. 166-180, 2015. 


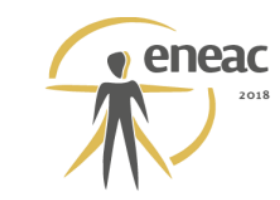

RHEINGANTZ, P. A.; ALCANTARA, Denise de; RIO, Vicente del. A influência do projeto na qualidade do lugar: Percepção da Qualidade em Áreas Residenciais do Rio de Janeiro.

Sociedade e Território - Revista de estudos urbanos e regionais - \# 39. Rio de Janeiro, dezembro 2005.

SHEHAB, E.; BOUIN-PORTET, M.; HOLE, R.; FOWLER, C. Enhancing digital design data availability in the aerospace industry. CIRP Journal of Manufacturing Science and Technology, v. 2, n. 4, p. $240-246,2010$.

UVA, A. E.; CRISTIANO, S.; FIORENTINO, M.; MONNO, G. Distributed design review using tangible augmented technical drawings. Computer-Aided Design, v. 42, n. 5, p. $364-372$, 2010.

VASCONCELOS, C. S. F e; VILLAROUCO, V.; SOARES, M. M. Avaliação ergonômica do ambiente construído: estudo de caso em uma biblioteca universitária. Ação ergonômica: revista brasileira de ergonomia, v.4, n.1, 2009.

WILSON, John R. Virtual environment applications and applied ergonomics. Applied Ergonomics, v.30, 1999, p.3-9. 